\title{
SRF Fixture Design and HOM analysis
}

\section{James Griggs, University of Kentucky}

\section{Background}

A particle accelerator can either be a normal conducting or a superconducting accelerator. While a superconducting accelerator is a more energy efficient option, they traditionally use a liquid helium bath to achieve the temperatures needed for superconductivity. This makes superconducting accelerators operationally complex and expensive. To make superconducting accelerators a more commercially viable option IARC is working on a cryogen free accelerator which can be seen below.

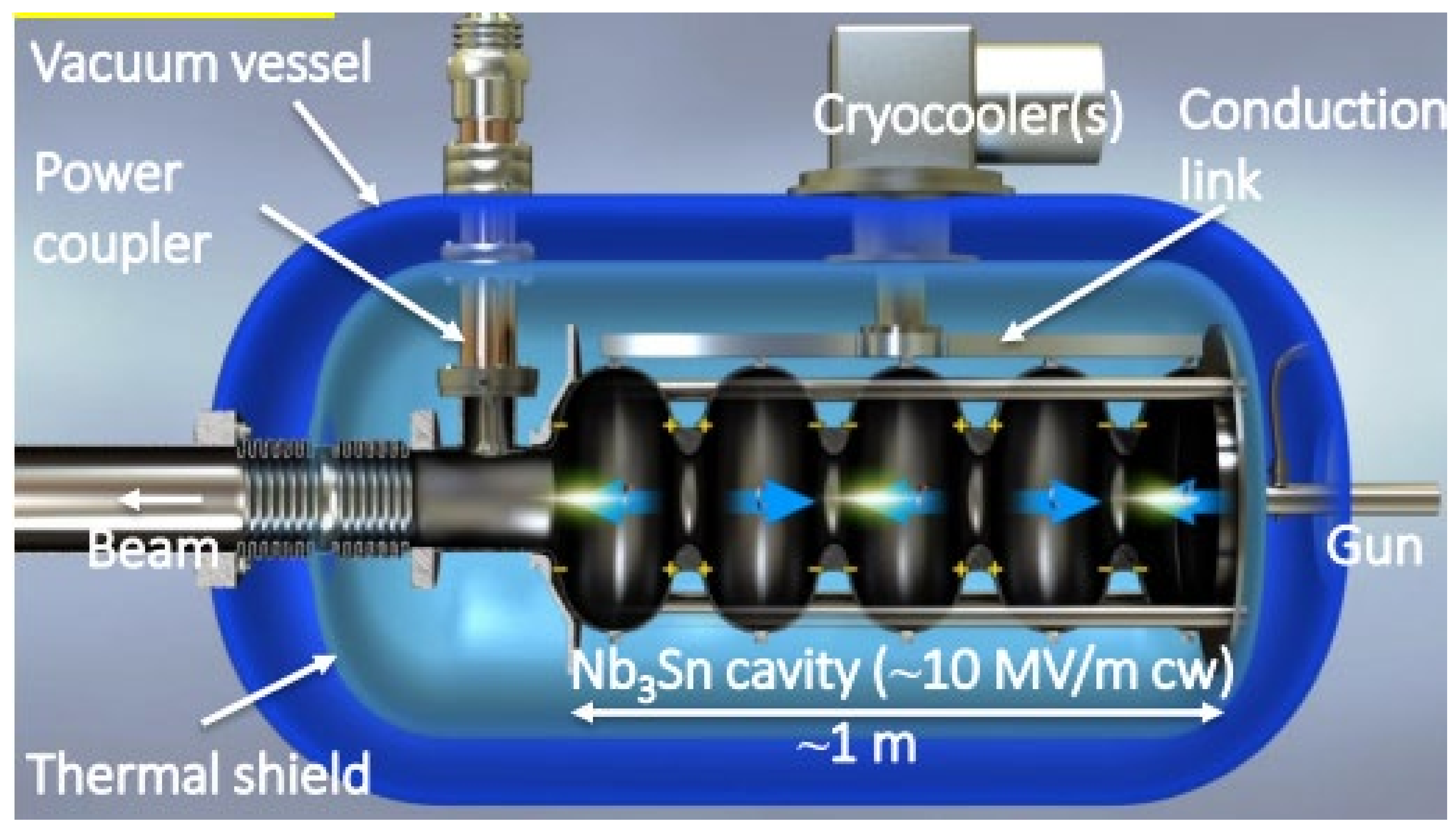

IARC's 4.5 cell Cryocooled superconducting accelerator.

IARC's accelerator achieve supercooling through use of Crycoolers that link to the RF cavity and pulls heat away. It's these RF cavities that accelerate charged particles and can be viewed as the engine of the accelerator. These cavities use AC power in the radio wave frequency to create electric fields inside the cavities.

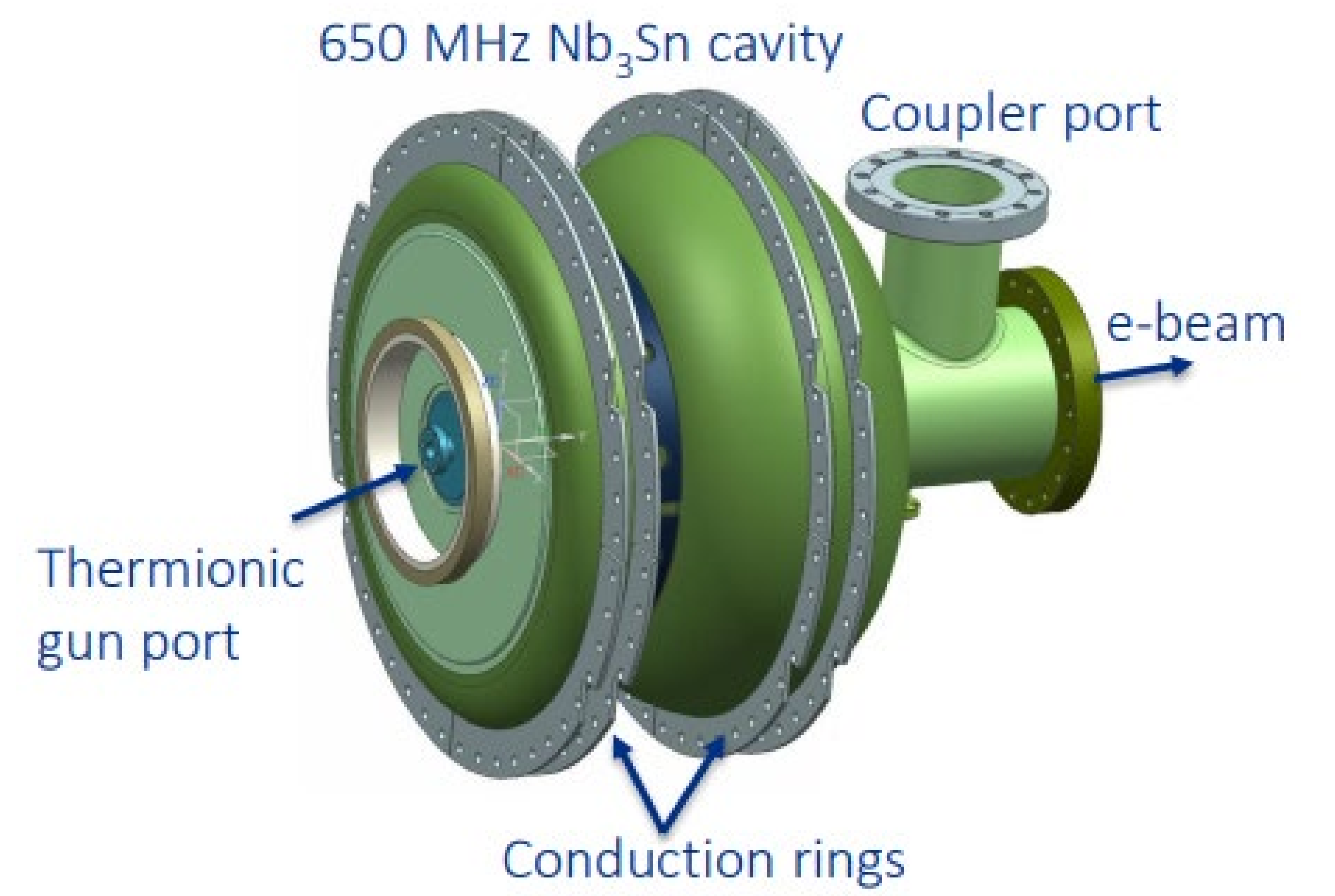

\section{Fixture Design}

During fabrication and quality testing, a cavity will go through many variations in pressure both internally and externally. The geometry of these cavities determines how electric fields develop inside the cavity and any small change can impede the cavity's ability to accelerate charged particles.

Since the cavity must have a vacuum pulled and then go through quality testing, a fixture is needed to help provide more rigidity during transport and additional support during the helium baseline test. A structure that can easily be attached and removed is needed.

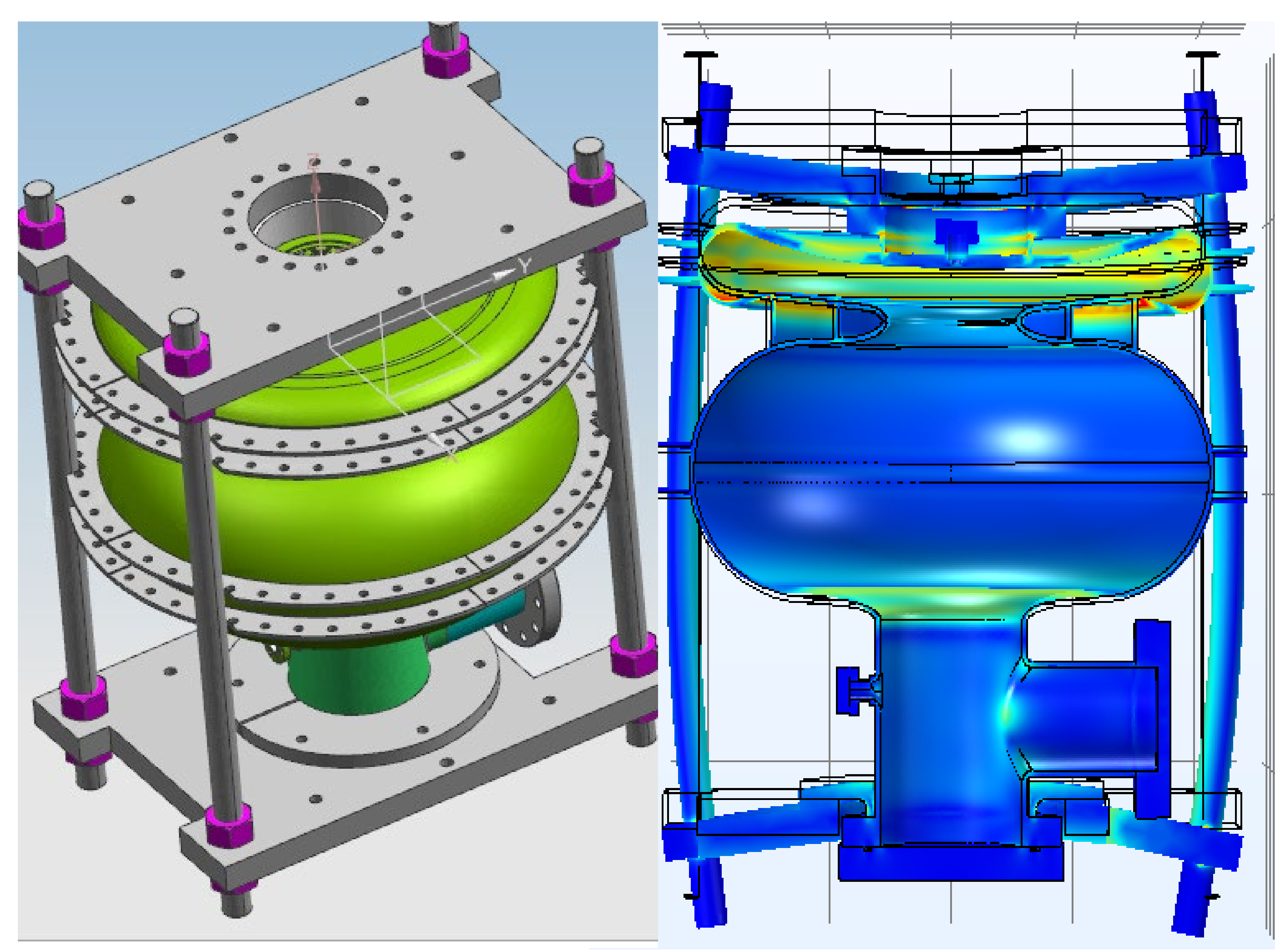

On the left is the CAD model as it was designed in NX11 and on the right is the model in COMSOL where FEA analysis was carried out to ensure the
structure would work as intended.

\section{HOM Analysis}

Since these cavities are designed to use standing waves to create strong electric fields, they can be viewed as electromagnetic resonate devices. The fundamental frequency of these cavities is what we want to use as the main accelerating mode. The issue is that these cavity will have an infinite number of resonate modes that wont hep accelerate charged particles, these modes are called Higher Order Modes (HOM).

The challenge in this cavity design is twofold, First is to make sure that HOM frequencies are not near the fundamental frequencies or its resonance frequencies, and the second is to make sure that any HOM near the fundamental or resonant frequencies has a low R/Q value. $R / Q$ can be viewed as a normalized shunt impedance and the amount of power stored in any mode is proportional to its $R / Q$ value.

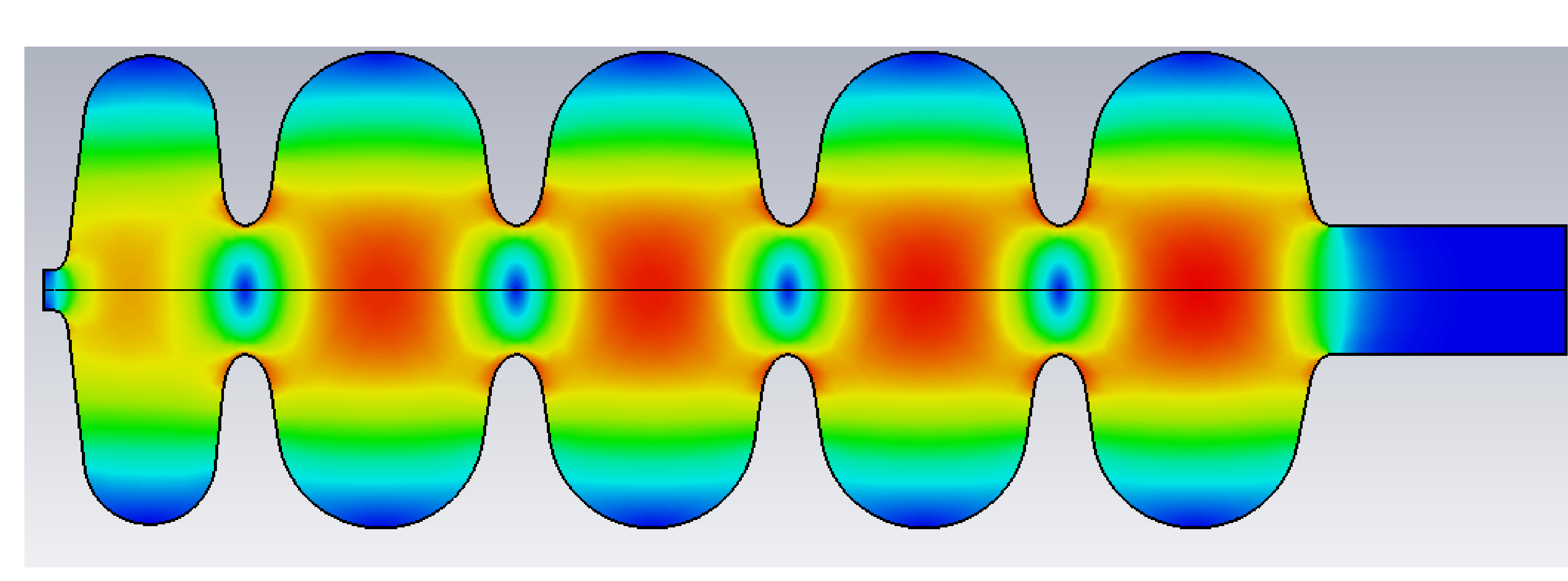

Simulations were carried out in CST: Microwave Studio found one monopole near the fundamental frequency that had a low enough R/Q to not be a concern. No monopole modes were found near higher harmonics.

Searching through HOM we found that for the 4.7 cavity geometry there were no monopole modes near higher order harmonic frequencies. 